\title{
Apports et limites d'une enquête internationale: le cas de PISA en Suisse romande
}

\section{Christian Nidegger}

L'enquête PISA est un projet international cyclique qui vise à mesurer les compétences des élèves de 15 ans. A travers l'expérience de la mise en ouvre de cette enquête dans un espace restreint (la Suisse romande), la publication des premiers résultats a eu un impact fort sur le monde de l'éducation en Suisse romande. La richesse, la quantité et la complexité des informations disponibles amène un certain nombre d'interrogations sur l'interprétation, la diffusion et l'utilisation des résultats. On se penchera également sur les problèmes que posent l'exploitation à un niveau local d'informations dont le recueil est conçu dans un but essentiellement de comparaison internationale.

L'enquête PISA ${ }^{1}$ (Programme international pour le suivi des acquis des élèves) pose la question de l'intrication et la confrontation de la réalisation de différents objectifs: relever un défi organisationnel c'est-à-dire réaliser régulièrement et dans un délai court (cycle de trois ans) l'ensemble des opérations nécessaires à la réalisation du projet, son exploitation et sa valorisation; satisfaire une visée politique en fournissant un instrument de comparaison de systèmes d'enseignement et de pilotage au niveau international ou national; enfin, relever un défi scientifique: utiliser à large échelle des méthodes de construction d'instruments, de recueil et d'analyses de données sophistiquées correspondant aux canons scientifiques actuels dans ce domaine.

Dans cet article, ces différents aspects sont mis en évidence à travers l'exemple de la mise en œuvre et réalisation de l'enquête PISA en Suisse romande. Ainsi, PISA a permis de développer une coopération entre les différentes institutions de recherches et d'acquérir un certain nombre de compétences dans le domaine des enquêtes à large échelle. Cela a également donné l'occasion d'être confronté aux questions que soulève l'exploitation locale d'une enquête internationale. Ces aspects seront décrits dans une première partie. Ensuite, les principaux résultats des cantons de la Suisse romande seront rappelés. Les apports et les limites de l'analyse et de l'interprétation des résultats seront présentés, notamment au niveau de leur pertinence locale ou internationale. Dans une dernière partie, seront 
esquissées les perspectives d'analyses secondaires en cours ou à venir et les liens possibles de PISA avec d'autres travaux.

\section{PISA, confronté à un défi organisationnel}

Rappelons que «le Programme international de l'OCDE pour le suivi des élèves (PISA) est une initiative concertée des pays de l'OCDE qui vise à évaluer dans quelle mesure les jeunes adultes de 15 ans [...] sont préparés à relever les défis de la société de la connaissance» (OCDE, 2001, p. 14). Ce programme s'appuie sur un certain nombre de principes. Nous en soulignerons trois:

- son orientation politique: le cadre conceptuel et les méthodes de compte rendu de l'évaluation ont été choisis pour permettre aux gouvernements de tirer des enseignements politiques des résultats;

- sa focalisation sur une manifestation des connaissances et des compétences sous une forme qui est pertinente pour la vie de tous les jours;

- son principe de collaboration: le programme est dirigé conjointement par les gouvernements des pays participants et bénéficie de l'expertise scientifique d'un consortium international constitué des plus éminents instituts spécialisés dans l'évaluation. (OCDE, 2001, p. 18).

Lors des travaux réalisés pour le premier cycle PISA, le consortium romand PISA $^{2}$, qui est chargé de l'enquête en Suisse romande, a rencontré un certain nombre de difficultés. La complexité du dispositif avec l'interpénétration des différents niveaux, cantonal, régional, national et international, crée une tension entre les objectifs ou les intérêts des partenaires de ces différents niveaux. Par exemple, lorsqu'il s'agit d'adapter les normes et les instructions internationales, une bonne solution au niveau national n'est pas toujours pertinente au niveau régional ou cantonal. Ceci est particulièrement vrai en Suisse où la présence de structures différentes des systèmes scolaires rend difficile la rédaction de questions portant sur la description du système scolaire qui soient compréhensibles et pertinentes pour l'ensemble des destinataires du questionnaire, élèves ou direction d'écoles et qui permettent en même temps la comparaison entre les systèmes cantonaux. De plus, par la nature internationale de l'enquête, l'impact du niveau local sur les décisions internationales est faible.

Une difficulté supplémentaire provient de la confrontation permanente de deux logiques dans le projet: une logique "administrative» et organisationnelle nécessaire à la réalisation et à la mise en place concrète de l'enquête et qui vise à assurer une cohérence, pour ne pas dire une uniformité des instruments et des conditions de passation et de récolte des informations. D’autre part, une démarche scientifique qui cherche à mettre en évidence des différences par l'utilisation d'instruments et de procédures sans toujours tenir compte des problèmes techniques, des contraintes imposées par le fonctionnement et l'organisation des écoles dans lesquelles nous devons intervenir. L'expérience de la mise en œuvre 
de PISA en Suisse a montré l'utilité d'une décentralisation maximum des tâches nécessaires à la mise en œuvre de l'enquête au niveau des régions linguistiques afin de «coller» le plus possible à la réalité du terrain et d'assurer un optimum entre exigences internationales et possibilités d'adaptation locale.

\section{PISA, source d'un débat politique}

La publication des premiers résultats de PISA a suscité de fortes réactions en Suisse et particulièrement en Suisse romande. C'est très certainement une des premières fois qu'une enquête scientifique dans le domaine de l'éducation a déclenché autant de commentaires, prises de position, débats, analyses auprès des différents milieux de l'éducation. Chacun y est allé de son appréciation ou a convoqué son ou ses experts à même de disséquer et d'interpréter les données présentées, voir à ce propos l'article de Weiss (2002) sur la réception de l'enquête par les différents groupes d'acteurs.

Du point de vue de l'impact de l'enquête en Suisse et en Suisse romande, on peut dire que l'objectif est pleinement atteint. On peut cependant se poser la question des raisons qui font que la publication de ces résultats ait eu un tel impact. On notera que dans d'autres pays l'impact auprès du public et même des groupes d'acteurs proches de l'éducation, a été faible voire nul par exemple en Italie ou en France. En Suisse et notamment en Suisse romande, on peut dire que PISA est un élément incontournable de toute discussion ayant trait à l'éducation même si l'objet de la discussion est très éloigné d'éléments mesurés par PISA. Quels sont donc les principaux résultats de PISA? Comment peuvent-ils être interprétés? Quels liens entre comparaison internationale et locale? Quels problèmes cela pose-t-il? Et quelle suite donner à PISA? Quelles autres exploitations des données peut-on encore mener?

\section{PISA, un défi scientifique à relever}

\section{Une méthodologie complexe}

La méthodologie utilisée dans PISA est complexe. Pour couvrir les champs de compétence les plus larges possibles, un grand nombre de questions sont proposées aux élèves. On ne peut soumettre tous les élèves à l'ensemble de ces questions qui correspondraient à un test d'une durée totale de 7 heures. Pour réduire la passation à une durée d'environ 2 heures, l'ensemble des items prévus sont répartis dans différents cahiers distribués aléatoirement aux élèves. Les élèves ne sont donc pas tous confrontés aux mêmes questions. Pour évaluer les performances des élèves, on ne peut pas se contenter de dénombrer les bonnes réponses. Une telle approche conduirait à une évaluation incorrecte des performances. En effet, les questions sont de difficulté variable et les élèves ne passent pas tous 
le même test. Pour être en mesure de construire des échelles valables pour tous, on doit s'appuyer sur une autre approche (celle de la théorie de réponse aux items). Cette approche décrit la relation entre le niveau de compétence d'un élève et la probabilité de réponse correcte à un item. Elle permet de situer sur une même échelle la difficulté des items et les compétences des élèves. Pour chaque domaine, on peut alors comparer les items entre eux par leur niveau de difficulté et les élèves en fonction de leur score sur cette échelle. Cette démarche va parfois contre le sens commun ou les habitudes d'évaluation pratiquées notamment dans le cadre scolaire où habituellement on comptabilise le nombre de réponses justes de chaque élève à une épreuve commune à la population que l'on désire évaluer. Cette distance peut donc être un obstacle pour la compréhension et l'interprétation des résultats de PISA. Elle peut être aussi source de tension et de méfiance de la part du monde enseignant. On peut notamment regretter que les enseignants soient peu associés dans les processus PISA. Les contraintes des modèles d'analyse utilisés semblent actuellement faire que ceux-ci restent aux mains d'un groupe d'experts limité alors que ces instruments utilisés visent à mesurer, d'une certaine manière le monde de l'enseignement. Dans ce contexte, ne devrait-on pas se poser la question d'une diversité et d'une complémentarité des méthodes à utiliser dans un processus complexe d'une grande enquête internationale?

De quelques problèmes attendus ...

En Suisse, avec la publication des premiers résultats de l'enquête réalisée en 2000 (OFS/CDIP, (2002) et Nidegger, (2001)), on pouvait avoir l'impression que soudain la Suisse et la Suisse romande en particulier découvraient que son école n'était ni la meilleure du monde ni la plus équitable. Cependant, ces résultats confirment ce qui avait déjà été mis en évidence dans d'autres études (Reading Literacy, 1991; TIMSS, 1995) à savoir des résultats relativement moyens en littératie et en sciences et des performances largement au-dessus de la moyenne des pays de l'OCDE en ce qui concerne les mathématiques. Les variations entre cantons sont en moyenne peu élevées. Par contre, les contextes cantonaux sont contrastés du point de vue des caractéristiques de leurs populations: genre, langue parlée, origine de la famille, niveau socio-économique ou de leur organisation scolaire: existence de filières et nombre de celles-ci. Une première analyse des données montre que seul un faisceau de variables peut expliquer ces différences et qu'il ne faut pas tenter d'attribuer à une cause unique les écarts constatés au niveau des performances. 
Tableau 1: Pourcentage des élèves dont un des membres de la famille n'est pas né en Suisse et résultats moyens en lecture

\begin{tabular}{|l|c|c|}
\hline & $\begin{array}{c}\text { un membre de la } \\
\text { famille n'est } \\
\text { pas né en Suisse }\end{array}$ & $\begin{array}{c}\text { moyenne en } \\
\text { lecture }\end{array}$ \\
\hline GE & 73.5 & 479 \\
\hline NE & 46.5 & 497 \\
\hline VD & 45.6 & 498 \\
\hline VS & 38.6 & 518 \\
\hline JU & 30.8 & 490 \\
\hline FR & 29.7 & 526 \\
\hline Suisse romande & 48.2 & 499 \\
\hline
\end{tabular}

On remarquera par exemple dans le tableau ci-dessus que la proportion d'élèves qui ne sont pas nés en Suisse est importante à Genève où les résultats moyens sont les plus faibles. Par contre, le Jura qui a des résultats proches de Genève a un taux relativement faible d'élèves qui ne sont pas nés en Suisse

Tableau 2: Résultats en LECTURE dans les différents cantons romands: répartition par niveau de compétences en pourcentage

\begin{tabular}{|l|c|c|c|c|c|c|}
\hline & Fribourg & Genève & Jura & Neuchâtel & Valais & Vaud \\
\hline niveau 0 & 1.2 & 8.0 & 1.1 & 3.3 & 0.9 & 3.9 \\
\hline niveau 1 & 4.6 & 13.2 & 12.3 & 11.5 & 4.2 & 9.2 \\
\hline niveau 2 & 17.5 & 27.1 & 31.4 & 23.6 & 21.8 & 25.3 \\
\hline niveau 3 & 41.7 & 29.3 & 32.4 & 35.8 & 41.8 & 37.0 \\
\hline niveau 4 & 28.7 & 18.8 & 19.6 & 21.1 & 26.8 & 18.9 \\
\hline niveau 5 & 6.3 & 3.5 & 3.2 & 4.6 & 4.5 & 5.8 \\
\hline
\end{tabular}

Un point central est à souligner: l'existence d'une frange non négligeable d'élèves qui à la fin de la scolarité obligatoire ont des compétences très limitées en littératie: entre $5 \%$ (Valais) et $21 \%$ (Genève) des élèves se situent au niveau 1 ou endessous. Ces résultats risquent d'avoir des conséquences sur leur réussite scolaire dans les autres matières scolaires, leur scolarité en général et sur leur avenir professionnel. Ceci est une interrogation très importante faite à nos systèmes scolaires en termes qualitatifs car cela signifie que ces élèves n'ont pas réussi à tirer un bénéfice suffisant de leur scolarité malgré leur longue présence à l'école. 
Tableau 3: Pourcentages des élèves présents ou absents lors $d u$ test par canton

\begin{tabular}{|l|c|c|c|}
\hline & présent & exclu & absent \\
\hline FR & $94.0 \%$ & $2.9 \%$ & $3.0 \%$ \\
\hline GE & $90.5 \%$ & $0.4 \%$ & $9.2 \%$ \\
\hline JU & $95.5 \%$ & $0.5 \%$ & $4.0 \%$ \\
\hline NE & $95.1 \%$ & $0.0 \%$ & $4.9 \%$ \\
\hline VS & $94.7 \%$ & $0.3 \%$ & $4.9 \%$ \\
\hline VD & $89.9 \%$ & $0.5 \%$ & $9.7 \%$ \\
\hline Suisse romande & $93.3 \%$ & $0.8 \%$ & $5.9 \%$ \\
\hline
\end{tabular}

Les résultats présentés jusqu'à maintenant se réferent aux performances des élèves au test ou aux informations recueillies à travers le questionnaire aux élèves. Un élément supplémentaire nous semble important à prendre en compte c'est le contexte dans lequel se déroule la passation. Ainsi, la part des élèves qui ne participent pas au test peut nous donner des indications sur ce contexte. Le tableau ci-dessus indique le pourcentage d'élèves exclus ${ }^{3}$ de l'enquête selon les critères internationaux (handicap fonctionnel ou mental, connaissance insuffisante du français) ou absents le jour du test. Si la proportion d'élèves exclus est très faible, on constate une variation relativement importante des élèves absents entre les cantons: moins de $5 \%$ dans tous les cantons sauf sur Vaud et Genève où ce taux se situe au-delà de $9 \%$. On pourrait faire l'hypothèse que ce taux assez élevé s'explique en partie par un rapport plus distancié à l'institution scolaire. En effet, pour ces deux cantons, qui sont les plus urbanisés, l'école n'est peut-être pas un élément central de la vie sociale. Les valeurs et les fonctions que les différents groupes d'acteurs attribuent à l'école sont plus contrastées et diversifiées.

Ces quelques résultats font ressortir la richesse et la diversité des informations que peut fournir PISA. Mais ces résultats illustrent également la difficulté d'interpréter ces données de façon unique et la nécessité de transmettre un message qui met en perspective la complexité des réponses possibles aux problèmes ou questions que soulèvent PISA.

... et de quelques subtilités d'interprétation

Voyons maintenant quelques problèmes que l'on peut rencontrer lorsqu'on analyse les données selon plusieurs perspectives: internationale, nationale ou régionale.

Rappelons d'abord que la visée de PISA est essentiellement comparative. Cette comparaison n'est pas envisagée dans la perspective de la publication de classement des pays mais dans le but de disposer d'éléments à même de déterminer les caractéristiques qui font que certains pays réussissent mieux que d'autres. 
Le projet est ambitieux car malgré ces affirmations, on remarque que la présentation du classement des pays ou des régions est une pratique que l'on a rencontrée très souvent bien que les concepteurs de l'enquête insistent sur le caractère secondaire de ces résultats. En effet, il est indiqué dans le rapport international initial (OCDE, 2001) en présentation de la comparaison des différences de moyennes entre pays

Il y a toutefois lieu de garder présent à l'esprit le fait que les chiffres de performance moyenne occultent des variations significatives au sein d'un même pays, [...] seul un dixième de la variation totale des performances des élèves enregistrée par le PISA renvoie à des différences entre pays [...] (p. 54).

Ainsi, l'analyse de ce qui se passe à l'intérieur de chaque pays est en tout cas aussi importante que ce que l'on peut retirer de la comparaison entre les pays. Du point de vue de la comparaison, on note que dans le rapport international, des précautions sont prises pour attirer l'attention du lecteur, dans certains cas, sur les limites de ces comparaisons. Ainsi, au sujet de l'exploitation du questionnaire aux écoles, on peut lire dans ce même rapport:

Il convient dans l'interprétation de ces chiffres, de garder à l'esprit le fait que les données recueillies auprès des chefs d'établissement sont limitées à certains égards. Dans la plupart des pays, 150 chefs d'établissement seulement ont répondu au questionnaire [...] La généralisation au départ d'une source d'information unique [...] est un procédé détourné (p. 174).

En effet, dans l'analyse des résultats romands, cette source d'information n'a quasiment pas été utilisée pour des comparaisons entre cantons car nous aurions eu une quantité insuffisante de mesures pour chaque canton. Dans certains cantons, le nombre total d'écoles concernées étaient de l'ordre d'une quinzaine. Plus généralement, on peut se poser la question de la validité de certaines comparaisons lorsque l'on passe de la comparaison entre pays à celle d'entités à l'intérieur d'un pays, comme par exemple les cantons. En effet, le processus de construction des instruments de l'enquête ${ }^{4}$ cherche à rendre possible la comparaison entre pays. Pour les tests cognitifs, on a veillé à disposer d'un matériel reflétant la diversité culturelle et linguistique des participants en mettant en place un dispositif de double traduction et d'adaptation qui évite le plus possible l'apparition de biais culturel ou linguistique ${ }^{5}$. Ce souci est également présent pour les informations recueillies à travers les questionnaires aux élèves ou aux écoles. Ainsi, on a recouru aux classifications internationales usuelles pour catégoriser les systèmes de formation des pays ou les professions des parents. Cependant, si toutes ces précautions dans la réalisation du matériel et le recours à des classifications reconnues facilitent la comparaison, elles ne sont pas toujours adéquates ou assez fines lors d'analyses locales.

Habituellement, on observe une relation entre le niveau socio-économique et la performance des élèves, ceci s'observe également dans l'enquête PISA. Lorsque l'on compare la moyenne du niveau socio-économique des différentes sections ou filières des cantons, on constate que les filières ou sections les plus valorisées 
ont un niveau socio-économique moyen plus élevé. Ceci se vérifie dans tous les cantons. Par contre, lorsqu'on observe les moyennes de l'indice du niveau socioéconomique dans les différents cantons, on constate que le canton de Genève a la moyenne la plus élevée et se distingue de tous les autres cantons alors qu'il obtient les compétences moyennes les plus basses. Le Jura a la moyenne la plus basse pour le niveau socio-économique avec des compétences moyennes comparables à celles de Genève. Le constat fait à l'intérieur de chaque canton ne se confirme pas entre les cantons. Ceci conforte l'idée que les différences de performances ne peuvent pas être attribuées à une seule variable et incite à une lecture des résultats à plusieurs niveaux. Pour cet indice du niveau socio-économique, on pourrait également faire l'hypothèse d'une distorsion de l'indice qui «favorise» les professions des services en attribuant une valeur d'indice plus élevé à ces professions qu’à des professions «artisanales» qui exigent des qualifications égales ou supérieures.

Lors de l'analyse des résultats de la Suisse et de la Suisse romande, on se trouve confronté à un obstacle supplémentaire. En effet, les populations choisies pour les comparaisons entre régions linguistiques ou entre cantons concernent des élèves de 9ème quel que soit leur âge, alors que l'enquête internationale porte sur les élèves de 15 ans quelle que soit leur place dans le système scolaire. La tentation est grande de comparer directement ces deux groupes par exemple en plaçant dans un même graphique le classement, selon les moyennes de performances, les régions ou les cantons avec les pays alors que le recouvrement entre ces deux populations peut être relativement différent selon les régions. En Suisse alémanique, $65 \%$ des élèves de 15 ans sont en 9ème alors qu'ils ne sont que $50 \%$ en Suisse romande et seulement $28 \%$ en Suisse italienne car l'âge d'entrée à l'école varie d'une région à l'autre. Ceci peut amener des différences relativement importantes pour une même entité selon qu'on considère les 15 ans ou les élèves de 9ème et cette différence pourra être influencée par la structure des entités considérées. Ainsi, la Suisse italienne, dont une partie importante de sa population de 15 ans se trouve dans le secondaire II, obtient un score moyen en lecture plus élevé de plus de 10 points si l'on considère sa population de 15 ans et non les élèves de 9 ème.

\section{Une recherche encore incomplète}

L'exploitation des données recueillies dans le cadre de PISA ne fait que commencer. La publication des premiers résultats internationaux, nationaux ou régionaux n'est qu'une première étape. Un certain nombre d'études complémentaires aux différents niveaux, international, national, régional sont en cours afin d'approfondir les résultats et d'exploiter le matériel récolté. Par exemple, la Suisse romande est impliquée, dans une étude qui vise à approfondir la connaissance des compétences en littératie (compréhension de l'écrit) notamment en lien avec 
le type de textes, la forme du questionnement et les programmes d'enseignement. Deux autres études sont en cours, l'une traitant de l'apprentissage auto-régulé son lien avec les performances et le contexte familial et scolaire et l'autre abordant l'impact des nouvelles technologies, leurs liens avec les compétences en littératie. PISA, par les réactions qu'il a suscitées, a également un effet indirect sur d'autres travaux, c'est d'ailleurs un des buts de ce genre de recherche, poser des questions dont les réponses ne se trouvent pas nécessairement dans les données PISA mais demandera d'autres récoltes d'information et d'autres investigations. Par exemple en Suisse romande, la publication des résultats de PISA a été l'occasion de souligner l'importance des travaux en cours autour du plan d'études cadre romand (PECARO). A Genève a été mis sur pied un "groupe opérationnel-français» chargé de la mise en œuvre d'un "plan d'action en faveur de la coordination de l'apprentissage du français durant la scolarité obligatoire». (CapCO Correspondances, octobre 2002). Cependant, le rythme soutenu des cycles de PISA (trois ans) permet-il de conduire ces études sans précipitation et mûre réflexion? On n'a pas encore exploité l'ensemble des données de PISA 2000 que déjà se profile le deuxième cycle de l'enquête. On risque ainsi de disposer de beaucoup d'informations mais de peu temps pour les analyser et les interpréter.

\section{Conclusion}

Quels sont les apports et les limites de PISA en Suisse romande au terme des quelques éléments présentés ci-dessus? Du point de vue organisationnel, la participation de notre région a permis de bénéficier de la planification et des structures mises en place aux niveaux international et national pour la réalisation de l'enquête. En contrepartie, cela a nécessité un investissement important de ressources financières, administratives et scientifiques afin de mener à bien sa mise en œuvre et surtout son exploitation. L'expérience acquise lors du premier cycle devrait nous amener à mieux étudier la question de la place que devrait prendre ce type d'enquêtes par rapport à d'autres dispositifs de recueils d'information afin de créer des synergies entre PISA et ces autres sources d'informations sur nos systèmes scolaires.

Au niveau politique, PISA a suscité de nombreux débats et une large utilisation par les différents acteurs de l'école de la masse importante d'informations riches, encore que partiellement exploitées, du premier cycle PISA. Face à cette quantité de données, il s'agit d'éviter de considérer PISA comme la source des réponses à toutes les questions relevant de l'éducation. Elle n'est qu'un élément constitutif de l'ensemble des connaissances dont nous disposons sur l'école et son environnement. Une des dérives de l'utilisation des résultats de PISA serait de réduire ces résultats à un catalogue dans lequel chacun choisit les interprétations qui sont le plus en phase avec sa vision de l'école ou ses intentions de changements. Il est dès lors important que les chercheurs rappellent que ces informa- 
tions ne prennent sens que dans le contexte dans lequel elles s'insèrent. Il faut donc lire et interpréter les résultats à la lumière de ces contextes.

Du point de vue scientifique, PISA a été l'occasion, pour les personnes qui ont participé à la réalisation de l'enquête en Suisse romande, d'acquérir de l'expérience et des connaissances dans le domaine de la réalisation d'enquêtes à large échelle. Certes, la démarche utilisée est complexe et les méthodes statistiques peuvent parfois faire écran et paraître abstraites. La complexité des modèles utilisés pour le traitement des données rend la lisibilité des interprétations difficile et ne favorise pas l'appropriation des résultats par les partenaires de l'école (autorités scolaires, direction d'établissements, formateurs, enseignants), créant ainsi un fossé entre eux et les «experts» à même de décoder et d'expliquer les résultats.

Pour les chercheurs, lorsqu'ils traitent les données à un niveau local, il importe qu'ils se posent la question de la pertinence des instruments, conçus pour une comparaison à large échelle, pour une exploitation locale afin d'éviter des interprétations abusives ou incohérentes des résultats observés. Ceci implique donc de disposer de personnes compétentes à même de maîtriser les instruments et les méthodes utilisées par PISA tout en connaissant bien le contexte et les spécificités des systèmes éducatifs locaux afin de donner du sens à l'exploitation locale de ces résultats. La prise en compte des contextes locaux ne peut que conduire à une amélioration de la qualité globale de ces enquêtes.

Finalement, une «bonne» exploitation au niveau régional d'une enquête telle que PISA dépendra de la prise en compte des différentes dimensions; organisationnelle, politique et scientifique que comportent un tel projet, de leur analyse critique, et de la mise en relation de ces différents aspects. Ceci afin que l'ensemble permette de dégager des résultats significatifs et cohérents du point de vue scientifique, contextualisés en fonction des niveaux d'analyse concernés (régional, national, international) et compréhensibles pour les partenaires de l'école.

\section{Notes}

1 En Suisse PISA est pilotée conjointement par la Confédération et les cantons par l'intermédiaire de la conférence des Chefs des départements de l'instruction publique (CDIP). La direction nationale de l'enquête a été confiée à l'Office fédéral de la Statistique (OFS).

2 A l'initiative des centres cantonaux de recherche et notamment du Service de la recherche en éducation (SRED) et de l'Institut de recherche et de documentation pédagogique (IRDP), un consortium romand pour l'évaluation des acquis et des compétences des élèves a été constitué afin de réaliser l'enquête, exploiter et valoriser les données dans l'espace romand.

3 Le taux élevé d'exclus à Fribourg s'explique par le fait que les élèves qui ont suivi leur scolarité dans la partie germanophone du canton ont la possibilité d'effectuer une 10e année de scolarité dans une école francophone.

4 Le matériel d'enquête comprenait neuf cahiers de test pour l'élève (chaque élève se voyait attribué aléatoirement un des neuf cahiers), un questionnaire à l'élève et un questionnaire pour l'école. 
5 Plus de 50\% du matériel de l'enquête est d'origine anglo-saxonne. Cependant les résultats des pays anglo-saxons ne sont pas homogènes et on constate également une grande variation des résultats entre les différents pays ou communautés francophones.

Références bibliographiques

CapCO/Correspondances (2002), PISA 2000, et après? Genève, DIP.

Nidegger, Ch. (coord. (2001). Compétences des jeunes romands - Résultats de l'enquête PISA 2000 auprès des élèves de $9 e$ année. Neuchâtel: IRDP.

OCDE (2001). Connaissances et compétences: des atouts pour la vie - Premiers résultats de PISA 2000. Paris: OCDE.

OFS/CDIP (2002). Préparés pour la vie? Les compétences de base des jeunes - Rapport national de l'enquête PISA 2000. Pilotage du système de formation. Neuchâtel \& Berne: OFS/ CDIP.

Weiss, J. (2002). Pisa, ce que chacun y a vu. L'éducateur, (9).

\section{Möglichkeiten und Grenzen einer internationalen Erhebung am Beispiel von PISA in der französischsprachigen Schweiz}

\section{Zusammenfassung}

Die PISA-Erhebung strebt an, international vergleichend und wiederkehrend die Kompetenzen der 15-jährigen Schülerinnen und Schüler zu messen. Nach den Erfahrungen der Umsetzung dieser Erhebung in einem begrenzten geografischen Raum (französischsprachige Schweiz) hat die Publikation der ersten Resultate eine starke Wirkung in der pädagogischen Welt der französischsprachigen Schweiz erzeugt. Der Reichtum, die Quantität und die Komplexität der verfügbaren Informationen führt jedoch zu Fragen hinsichtlich der Interpretation, Verbreitung und Verwendung der Resultate. Man wird sich auch mit den Problemen zu beschäftigen haben, die sich bei der lokalen Nutzung von Informationen, deren Erhebung vor allem auf einen internationalen Vergleich hin orientiert war, stellen.

\section{Apporti e limiti dell'utilizzazione dei dati di un'inchiesta internazionale: il caso PISA riferito alla Svizzera romanda}

Riassunto

L'inchiesta PISA è un progetto internazionale a carattere ciclico che mira alla valutazione delle competenze degli allievi di 15 anni. L'esperienza di uno spazio limitato (la Svizzera romanda) mostra come la pubblicazione dei primi risultati abbia avuto un impatto forte sul mondo dell'educazione. Ricchezza, quantità e 
complessità dei dati disponibili sono fattori che inducono alcuni interrogativi al riguardo dell'interpretazione, della diffusione e dell'utilizzazione dei risultati. Il contributo affronta pure la questione relativa all'utilizzazione a livello locale di informazioni finalizzate essenzialmente ad un confronto internazionale.

\section{Contributions and limitations of an international investigation. The case of the PISA study in French speaking Switzerland.}

\section{Summary}

The PISA study is a cyclical international project aimed at measuring the competency levels of 15 year olds. Through the experience of putting this study into action within a restricted geographical area (French speaking Switzerland), rather impressive reactions to the first results could be seen within the education community of French speaking Switzerland. The value, the quality and the complexity of the available information posses a certain number of questions about the data's interpretation, its distribution and its use. Problems concerning the examination of the results at different levels will also be looked at. The results were meant to be used in an international comparison and not at the local level. 